\title{
AN ALMOST SURE LIMIT THEOREM FOR MOVING AVERAGES OF RANDOM VARIABLES BETWEEN THE STRONG LAW OF LARGE NUMBERS AND THE ERDÖS-RÉNYI LAW
}

\author{
HARTMUT LANZINGER
}

\begin{abstract}
We prove a strong law of large numbers for moving averages of the form $(\log n)^{-p} \sum_{k=n+1}^{n+\left[(\log n)^{p}\right]} X_{k}$ when the moment condition $\mathrm{E}\left(\exp \left\{t\left|X_{1}\right|^{1 / p}\right\}\right)$ is imposed (with some $p>1$ ). It will turn out that due to the extreme terms among the $X_{k}$ these means do not satisfy a strong law in the classical sense but we can identify its upper and lower limit.
\end{abstract}

\section{INTRODUCTION}

In this paper we intend to close a gap appearing in the theory of strong limit theorems for moving averages of random variables. To start with we recall the classical strong law of large numbers due to Kolmogoroff $(1930,1933)$ : TheOREM K Let $X,\left(X_{k}\right)_{k=1}^{\infty}$ be independent, identically distributed random variables.

Then the sequence $\frac{1}{n} \sum_{k=1}^{n} X_{k}$ converges almost surely as $n \rightarrow \infty$ if and only if $\mathbf{E}|X|<\infty$.

In this case the a.s. limit equals $\mu=\mathbf{E} X$.

One of the many ways to generalize this result involves so called moving averages of random variables. In this context moving averages are means of the form $b_{n}^{-1} \sum_{k=n+1}^{n+b_{n}} X_{k}$ with a monotonically increasing sequence $\left(b_{n}\right)_{n=1}^{\infty}$, $b_{n} \in \mathbb{N}$ for all $n \in \mathbb{N}$ such that $b_{n} \rightarrow \infty \quad(n \rightarrow \infty)$. For moving averages there are well-known analytical results that relate strong laws for these means to strong laws for certain classes of weighted means (particularly summability methods). See e.g. Chow (1973) for such results concerning Euler methods, Bingham and Tenenbaum (1986) and Bingham and Goldie (1988) for corresponding theorems on Riesz means. Following this approach one can prove strong laws for certain summability methods if strong laws for suitable moving averages are given. The reader may consult Lai (1974), Chow (1973), Bingham and Tenenbaum (1986), Bingham and Maejima (1985) as well as Bingham and Stadtmïller (1990) for a large variety

The results of this paper form part of the author's dissertation written at the University of Ulm, Germany, under the guidance of Professor U. Stadtmüller.

URL address of the journal: http://www.emath.fr/ps/

Received by the journal February 25, 1997. Revised February 18, 1998. Accepted for publication September 17, 1998.

(C) Société de Mathématiques Appliquées et Industrielles. Typeset by $\mathrm{LATEX}_{\mathrm{E}}$ 
of results of that kind. Strong laws for very general classes of summability methods were obtained by Kiesel (1993). Of course this list does not aim at completeness. For a more complete account the reader is referred to Bingham $(1985,1988)$. The behavior of the sequence $\left(b_{n}^{-1} \sum_{k=n+1}^{n+b_{n}} X_{k}\right)_{n=1}^{\infty}$ differs from $\left(b_{n}^{-1} \sum_{k=1}^{b_{n}} X_{k}\right)_{n=1}^{\infty}$ as far as the covariance structures of the sequences are concerned. This covariance structure is crucial for almost sure convergence whereas from the point of view of convergence in probability there is no difference at all between both sequences. What can be said in general is that one has to impose the higher moment conditions the more slowly $\left(b_{n}\right)_{n=1}^{\infty}$ grows in order to obtain a strong law of large numbers. This can e.g. be seen from the following strong law for moving averages which is implicit in Chow (1973) and then is stated again in a more general framework by Bingham and Tenenbaum (1986):

Theorem C-BT Let $\left(X_{k}\right)_{k=1}^{\infty}$ be a sequence of independent, identically distributed random variables and $p>1$.

Then

if and only if

$$
\frac{1}{n^{1 / p}} \sum_{k=n+1}^{n+\left[n^{1 / p}\right]} X_{k} \rightarrow \mu \text { f.s. }
$$

$$
\mathbf{E}|X|^{p}<\infty \text { and } \mathbf{E} X=\mu \text {. }
$$

Similar results hold in situations with more general moment conditions such as $\mathbf{E} \psi(|X|)<\infty$ with functions $\psi$ more general than powers but with polynomial growth. For a theorem of this kind $\mathrm{cf}$. Bingham and Goldie (1988). This particular theorem applies to functions like $\psi(x)=x^{p}$ for some $p>1$ but not to $\psi(x)=e^{t x}$ for a $t>0$. It is not very surprising that some condition on the growth of $\psi$ is needed in order for this result to hold since Shepp (1964) proved the following:

Theorem S Let $X,\left(X_{k}\right)_{k=1}^{\infty}$ be independent, identically distributed random variables with $\mathbf{E} X=0$ and $M(t)=\mathbf{E} e^{t X}<\infty$ for all $t$ in a neighborhood of 0 .

Define $m(x)=\sup _{t \in \mathbb{R}}\{x t-\log M(t)\}$ for $x>0$. If $m(\gamma)=1 / c$ holds for $c$ and $\gamma$ then it follows:

$$
\limsup _{n \rightarrow \infty} \frac{1}{c \log n} \sum_{k=n+1}^{n+[c \log n]} X_{k}=\gamma \quad \text { a.s. }
$$

If $c$ varies in a suitable non-degenerate interval then the distribution of $X$ is uniquely determined by the limit.

Erdös and A. Rényi (1970) later proved a similar result, obviously unaware of Shepp's work.

Note that if the moment generating function of $X$ exists in an open neighborhood of 0 then there is a $c_{0} \in \mathbb{R}$ such that for every $c>c_{0}$ we may find some $\gamma \in \mathbb{R}$ with $m(\gamma)=1 / c$. Hence this condition is fulfilled at least for all $c \in\left(c_{0}, \infty\right)$. We may in particular let $c$ vary in an interval of positive length. 
We will denote such results as Erdös-Rényi-Shepp laws or Erdös-Rényi laws in the following. In contrast to the classical strong laws of large numbers we do not have invariance of the limit in this case. These topics also attracted a number of authors. We want to mention only Csörgö and J. Steinebach (1981), Steinebach (1978), Kiesel and Stadtmïller (1996), Deheuvels and Devroye (1987).

Comparing the above theorems one discovers that none of them applies to moving averages like $(\log n)^{-2} \sum_{k=n+1}^{n+\left[\log ^{2} n\right]} X_{k}$ under the moment condition $\mathbf{E}\left(\exp \left\{|X|^{1 / 2}\right\}<\infty\right.$. The function $\psi(x)=\exp \left\{x^{1 / 2}\right\}$ is not of polynomial growth so the result of Bingham and Goldie does not apply. But on the other hand we do not have any information about the moment generating function $\mathbf{E} e^{t X}$ that may not exist for any $t \neq 0$ in spite of the moment condition. So Theorem S does not apply either. Thus we can ask the question whether anything can be said about the behavior of moving averages like $(\log n)^{-2} \sum_{k=n+1}^{n+\left[\log ^{2} n\right]} X_{k}$ under the above moment condition, if a classical strong law or an Erdös-Rényi type law holds or if a third possibility applies. The answer to this question (in a more general form) is the main objective of this work. There are only few papers dealing with such averages. We only want to mention de Acosta and Kuelbs (1983) who partially examined such means in a very general setting (i.e. for random variables taking values in a separable Banach space).

\section{MAIN RESULTS}

From now on we assume without further comment that $X,\left(X_{k}\right)_{k=1}^{\infty}$ are independent, identically distributed random variables. We further set $g_{p}(x)=$ $\operatorname{sgn} x \cdot|x|^{1 / p}$ for $p \geq 1$ and $x \in \mathbb{R}$.

Theorem 2.1. For some $p>1$ we define $g(x)=g_{p}(x)$ and $a_{n}=(\log n)^{p}$. Further let $t_{1}, t_{2} \in(0, \infty]$.

Then the following are equivalent:

(i) $\mathbf{E} e^{\operatorname{tg}(X)}<\infty$ for $t \in\left(-t_{1}, t_{2}\right), \mathbf{E} e^{\operatorname{tg}(X)}=\infty$ for $t \notin\left[-t_{1}, t_{2}\right]$ and $\mathbf{E} X=$ $\mu$.

(ii) $\liminf _{n \rightarrow \infty} \frac{1}{a_{n}} \sum_{k=n+1}^{n+a_{n}} X_{k}=\mu-\frac{1}{t_{1}^{p}}$ a.s. and

$$
\limsup _{n \rightarrow \infty} \frac{1}{a_{n}} \sum_{k=n+1}^{n+a_{n}} X_{k}=\mu+\frac{1}{t_{2}^{p}} \text { a.s. }
$$

REMARK 2.2. Note that there might be different triples $\left(\mu, t_{1}, t_{2}\right)$ such that the respective values of $\mu+t_{2}^{-p}$ and $\mu-t_{1}^{-p}$ agree. Thus the statement of part (ii) $\Rightarrow$ (i) of the assertion is to be read as follows:

If $\liminf _{n \rightarrow \infty} a_{n}^{-1} \sum_{k=n+1}^{n+a_{n}} X_{k}$ and $\limsup _{n \rightarrow \infty} a_{n}^{-1} \sum_{k=n+1}^{n+a_{n}} X_{k}$ both are finite almost surely then $\mathbf{E} e^{t g(X)}<\infty$ in a neighborhood of 0 . Hence $\mathbf{E}|X|<$ $\infty$.

Setting $\mathbf{E} X=\mu$ we can now write $\liminf _{n \rightarrow \infty} a_{n}^{-1} \sum_{k=n+1}^{n+a_{n}} X_{k}$ as well as $\limsup _{n \rightarrow \infty} a_{n}^{-1} \sum_{k=n+1}^{n+a_{n}} X_{k}$ in the form $\mu-t_{1}^{-p}$ and $\mu+t_{2}^{-p}$, respectively, then the moment condition (i) holds.

Similar remarks also apply to all other results of this kind stated here. 
REMARK 2.3. Observe that the upper limit occuring in part (ii) of the assertion equals $\limsup _{n \rightarrow \infty} X_{n} / a_{n}$ which follows easily from the usual BorelCantelli argument. That means that the moving averages contain terms of the size of the norming constants again and again and that the moving averages become at most as large as these terms. This suggests that precisely these terms determine the non-classical behavior of these means which will be shown later. In the classical case such terms cannot occur because the moment conditions imposed on $X$ or $K X$ for arbitrary $K>0$ are equivalent. REMARK 2.4. The special case $t_{1}=t_{2}=\infty$ of Theorem 2.1 yields a strong law in the classical sense.

A similar proof also yields

Theorem 2.5. For some $p>1$ we define $a_{n}=(\log n)^{p}$.

Then the following are equivalent:

(i) $\mathbf{E} e^{t|X|^{1 / p}}<\infty$ for some $t>0, \mathbf{E} X=\mu$.

(ii)

$$
\begin{gathered}
\qquad \lim _{n \rightarrow \infty} \frac{1}{c_{n}} \sum_{k=n+1}^{n+c_{n}} X_{k}=\mu \\
\text { for every monotonically increasing sequence }\left(c_{n}\right)_{n=1}^{\infty} \text { with } c_{n} \geq 1 \text { and } \\
\frac{c_{n}}{a_{n}} \rightarrow \infty \quad(n \rightarrow \infty) .
\end{gathered}
$$

In Theorem 2.1 the upper and lower limits might differ from $\mathbf{E X}$. So we are in a situation that at least resembles the one of the law of the iterated logarithm. Like there one can also ask for the set of limit points of the sequence of moving averages in our setting. This question is answered by the next result. We denote here and later on the set of limit points of a given real sequence $\left(x_{n}\right)_{n=1}^{\infty}$ by $\mathbf{C}\left(\left\{x_{n}\right\}\right)$.

Theorem 2.6. For some $p>1$ we define $g(x)=g_{p}(x)$ and $a_{n}=(\log n)^{p}$. Further let $t_{1}, t_{2} \in(0, \infty]$.

Then the following are equivalent:

(i) $\mathbf{E} e^{t g(X)}<\infty$ for $t \in\left(-t_{1}, t_{2}\right), \mathbf{E} e^{\operatorname{tg}(X)}=\infty$ for $t \notin\left[-t_{1}, t_{2}\right]$ and $\mathbf{E} X=$ $\mu$.

(ii) $\mathbf{C}\left(\left\{\frac{1}{a_{n}} \sum_{k=n+1}^{n+a_{n}} X_{k}\right\}\right)=\left[\mu-\frac{1}{t_{1}^{p}}, \mu+\frac{1}{t_{2}^{p}}\right]$ a.s.

We now reconsider an aspect observed above. We have seen that the nonvanishing upper limit in Theorem 2.1 was a consequence of the largest terms occuring. This leads to the idea that it should be possible to prove a strong law in the sense of almost sure convergence to the mean under the moment conditions imposed above for moving averages slightly modified by excluding some terms with large modulus. This is done in Theorem 2.7.

Results of this kind were already shown by Mori $(1976,1977)$ for the classical strong law of large numbers and by Griffin $(1988,1988 \mathrm{a})$ for the law of the iterated logarithm with some ideas being due to Feller (1968). In these classical theorems the moment conditions $\mathbf{E}|X|<\infty$ or $\mathbf{E} X^{2}<\infty$, respectively, can be weakened by removing extremal terms. The methods we use in the sequel partially rely on techniques developed by Mori and Griffin. 
Theorem 2.7. For some $p>1$ we define $g(x)=g_{p}(x)$ and $a_{n}=(\log n)^{p}$. Further let $t_{1}, t_{2}>0$ be given.

We assume $\mathbf{E} e^{t g(X)}<\infty$ for all $t \in\left[-t_{1}, t_{2}\right]$ and $\mathbf{E} X=\mu$.

Finally let $\left(r_{n}\right)_{n=1}^{\infty}$ be a monotonically increasing sequence of positive integers such that $r_{n} \rightarrow \infty \quad(n \rightarrow \infty)$. Assume that there exists an $\alpha>1$ and a $\tau>0$ with

$$
\liminf _{n \rightarrow \infty} \frac{a_{n}}{r_{n}^{\alpha}(\log n)^{\tau}}>0
$$

Let $\sum_{k=n+1}^{n+a_{n}} X_{k}$ denote the sum $\sum_{k=n+1}^{n+a_{n}} X_{k}$ with the $r_{n}$ largest and the $r_{n}$ smallest terms excluded.

Then

$$
\frac{1}{a_{n}} \sum_{k=n+1}^{n+a_{n}} X_{k} \rightarrow \mu \text { a.s. } \quad(n \rightarrow \infty) .
$$

Remark 2.8. Condition (2.1) is satisfied e.g. by $r_{n}=(\log n)^{\gamma}$ with $\gamma \in$ $(0, p)$.

Theorem 2.9 shows that the condition $r_{n} \rightarrow \infty \quad(n \rightarrow \infty)$ of Theorem 2.7 is necessary. So there is no $r \in \mathbb{N}$ such that the moving averages without the $r$ largest and the $r$ smallest terms converge to the mean almost surely unless $\mathbf{E} e^{t|X|^{1 / p}} \operatorname{sgn} X<\infty$ for all $t \in \mathbb{R}$ in which case we have almost sure convergence to $\mathbf{E} X$ for the original moving averages already without removing any terms.

Theorem 2.9. For some $p>1$ we define $g(x)=g_{p}(x)$ and $a_{n}=(\log n)^{p}$. Further let $r \in \mathbb{N}$ as well as $t_{0}, t_{1}, t_{2}>0$ be given.

We assume $\mathbf{E} e^{t g(X)}<\infty$ for $t \in\left[-t_{1}, t_{0}\right]$ and $\mathbf{E} e^{t_{2} g(X)}=\infty$.

Finally let $\sum_{k=n+1}^{n+a_{n}} X_{k}$ denote the sum $\sum_{k=n+1}^{n+a_{n}} X_{k}$ with the $r-1$ largest summands removed. Then

$$
\limsup _{n \rightarrow \infty} \frac{1}{a_{n}} \sum_{k=n+1}^{n+a_{n}} X_{k} \geq \frac{1}{r^{p} t_{2}^{p}}
$$

REMARK 2.10. The proof of Theorem 2.9 shows that the r-1 smallest summands can be removed, too.

\section{Auxiliary RESUlts}

First we want to introduce some notation.

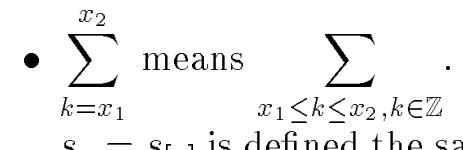

$s_{x}=s_{[x]}$ is defined the same way for an arbitrary real sequence $\left(s_{n}\right)_{n=0}^{\infty}$ and an arbitrary $x \geq 0$.

- The variable $C$ is supposed to represent a positive constant that may change within one sqeuence of inequalities.

- For $p>1$ we denote by $q$ the number $q>1$ with $p^{-1}+q^{-1}=1$. 
- We use the notations $L x=\max \{1, \log x\}, L L x=L(L x)$ and recursively define $L_{0} x=x$ and, for $\nu \in \mathbb{N}: L_{\nu} x=L\left(L_{\nu-1} x\right)$ (for $x \geq 0$, respectively). We further write for $\alpha>0: L^{\alpha} x=(L x)^{\alpha}$.

First we state a result that can be found in Lai (1974a).

LEMMA 3.1. Let $\left(Y_{k}\right)_{k=1}^{\infty}$ and $\left(Z_{k}\right)_{k=1}^{\infty}$ be two sequences of random variables on a probability space $(\Omega, \mathbf{F}, P)$ such that $\left(Y_{1}, Y_{2}, \ldots, Y_{n}\right)$ and $Z_{n}$ are independent for all $n \in \mathbb{N}$. Further let $a, b \in \mathbb{R}$ and $Z_{n} \stackrel{p}{\longrightarrow} b$ as $n \rightarrow \infty$.

Then the following holds:

a) If $\limsup \left(Y_{n}+Z_{n}\right) \leq a+b$ then $\lim \sup Y_{n} \leq a$ a.s.

b) If $\liminf _{n \rightarrow \infty}\left(Y_{n}+Z_{n}\right) \geq a+b$ then $\liminf _{n \rightarrow \infty} Y_{n} \geq a$ a.s.

We will also frequently use the well-known inequalities

$$
\begin{gathered}
1+x+\frac{x^{2}}{2} e^{-|x|} \leq e^{x} \leq 1+x+\frac{x^{2}}{2} e^{|x|}, \\
e^{x} \leq 1+x+\frac{x^{2}}{2} H(x)
\end{gathered}
$$

where $H(x)=\max \left\{1, e^{x}\right\}$ or simply

$$
1+x \leq e^{x}
$$

for $x \in \mathbb{R}$.

Next we state some easy technical lemmas.

Lemma 3.2. Let the functions $h_{k}:(0, \infty) \rightarrow(0, \infty) \quad(k=1,2)$ be monotonically increasing. Let $h_{1}(x) \rightarrow \infty \quad(x \rightarrow \infty)$ as well as $\frac{h_{2}(x)}{\left(h_{1}(x)\right)^{2}} \rightarrow 0 \quad(x \rightarrow$ $\infty)$.

Then we have for every $y \in \mathbb{R}$ :

$$
\left(1+\frac{y}{h_{1}(x)}\right)^{h_{2}(x)} \sim \exp \left\{y \frac{h_{2}(x)}{h_{1}(x)}\right\} \quad(x \rightarrow \infty) .
$$

Proof. It suffices to prove

$$
\log \left(1+\frac{y}{h_{1}(x)}\right)^{h_{2}(x)}-y \frac{h_{2}(x)}{h_{1}(x)} \rightarrow 0 \quad(x \rightarrow \infty) .
$$

But this follows easily by Taylor expansion of the logarithm.

The next lemma essentially contains, as a corollary, the Poisson approximation for sums of Bernoulli distributed random variables.

LEMMA 3.3. Let $\left(p_{n}\right)_{n=1}^{\infty}$ and $\left(r_{n}\right)_{n=1}^{\infty}$ be two sequences of numbers such that $p_{n} \in[0,1]$ and $n p_{n} \rightarrow 0(n \rightarrow \infty)$ as well as $r_{n} \in \mathbb{N}$ and $r_{n}^{2} / n \rightarrow 0(n \rightarrow \infty)$. Then

$$
\sum_{\nu=r_{n}}^{n}\left(\begin{array}{c}
n \\
\nu
\end{array}\right) p_{n}^{\nu}\left(1-p_{n}\right)^{n-\nu} \sim \frac{\left(n p_{n}\right)^{r_{n}}}{r_{n} !} \quad(n \rightarrow \infty) .
$$


Proof. We know

$$
\begin{aligned}
\sum_{\nu=r_{n}}^{n}\left(\begin{array}{c}
n \\
\nu
\end{array}\right) p_{n}^{\nu}\left(1-p_{n}\right)^{n-\nu} & \geq\left(n r_{n}\right) p_{n}^{r_{n}}\left(1-p_{n}\right)^{n-r_{n}} \\
& \geq \frac{n^{r_{n}}}{r_{n} !}\left(1-\frac{r_{n}}{n}\right)^{r_{n}} p_{n}^{r_{n}}\left(1-p_{n}\right)^{n} .
\end{aligned}
$$

From Lemma 3.2 we obtain

$$
\left(1-\frac{r_{n}}{n}\right)^{r_{n}} \sim \exp \left\{-\frac{r_{n}^{2}}{n}\right\} \rightarrow 1 \quad(n \rightarrow \infty)
$$

as well as

$$
\left(1-p_{n}\right)^{n} \sim e^{-n p_{n}} \rightarrow 1 \quad(n \rightarrow \infty)
$$

Furthermore

$$
\begin{aligned}
\frac{r_{n} !}{\left(n p_{n}\right)^{r_{n}}} \sum_{\nu=r_{n}}^{n}\left(\begin{array}{c}
n \\
\nu
\end{array}\right) p_{n}^{\nu}\left(1-p_{n}\right)^{n-\nu} & \leq \sum_{\nu=r_{n}}^{n} \frac{r_{n} !\left(n-r_{n}\right) !}{(n-\nu) ! \nu !} p_{n}^{\nu-r_{n}}\left(1-p_{n}\right)^{n-\nu} \\
& \leq \sum_{\nu=r_{n}}^{n} \frac{\left(n-r_{n}\right) !}{(n-\nu) !\left(\nu-r_{n}\right) !} p_{n}^{\nu-r_{n}}\left(1-p_{n}\right)^{n-\nu} \\
& =\sum_{\nu=0}^{n-r_{n}}\left(\begin{array}{c}
n-r_{n} \\
\nu
\end{array}\right) p_{n}^{\nu}\left(1-p_{n}\right)^{n-r_{n}-\nu}=1
\end{aligned}
$$

REMARK 3.4. The special case $r_{n}=r=$ const can e.g. be found in Mori (1976).

LEMMA 3.5. Let $Y$ be a random variable defined on a probability space $(\Omega, \mathbf{F}, P)$. Further let $a \in \mathbb{R}$ and $t_{1}, t_{2}>0$. Let $g(x)=g_{p}(x)$ for $x \in \mathbb{R}$.

Then

$$
\mathbf{E} e^{t g(Y)}<\infty \text { for all } t \in\left(-t_{1}, t_{2}\right)
$$

if and only if

$$
\mathbf{E} e^{t g(Y-a)}<\infty \text { for all } t \in\left(-t_{1}, t_{2}\right)
$$

Proof. It suffices to prove one direction. So assume $\mathbf{E} e^{t g(Y)}<\infty$ for all $t \in\left(-t_{1}, t_{2}\right)$.

Fix $t \in\left(0, t_{2}\right)$. If $a \geq 0$ then $g(Y-a) \leq g(Y)$ and the assertion follows immediately.

If $a<0$ the assertion follows from $Y-a \leq-a$ for $Y \leq 0$ and for $Y>0$

$$
Y-a=Y+|a| \leq\left(Y^{1 / p}+|a|^{1 / p}\right)^{p},
$$

i.e. $g(Y-a) \leq g(Y)+g(|a|)$.

The assertion for negative values of $t$ follows similarly.

Because we not only want to deduce a limit theorem from a moment condition but also vice versa we need a result that allows us to do this step. In that respect the following proposition is extremely useful. We use a notation matching the situation in later sections. 
Proposition 3.6. Let $g: \mathbb{R} \rightarrow \mathbb{R}$ be a strictly increasing function such that

$$
g(x) \rightarrow \infty \quad(x \rightarrow \infty) \text { and } g(x) \rightarrow-\infty \quad(x \rightarrow-\infty)
$$

For $a_{n}=g^{-1}(\log n)$ let

$$
\frac{a_{n}}{n} \leq K<\infty \text { for all } n \in \mathbb{N}
$$

Let

as well as

$$
\limsup _{n \rightarrow \infty} \frac{1}{a_{n}} \sum_{k=n+1}^{n+a_{n}} X_{k} \leq s_{0}
$$

$$
\liminf _{n \rightarrow \infty} \frac{1}{a_{n}} \sum_{k=n+1}^{n+a_{n}} X_{k} \geq-s_{1}
$$

with suitable constants $s_{0}>0$ and $s_{1}>0$.

Then $\mathbf{E}|X|<\infty$. If $\mathbf{E} X=0$ then we have also

$$
\mathbf{E} e^{g\left(\frac{1}{s} X\right)}<\infty
$$

for all $s>s_{0}$ and all $s<-s_{1}$.

Proof. Set $Y_{n}=\frac{1}{a_{n}} \sum_{k=n+1}^{n+a_{n}-1} X_{k}$ and $Z_{n}=\frac{X_{n+a_{n}}}{a_{n}}$. Note that $Z_{n} \stackrel{p}{\longrightarrow} 0 \quad(n \rightarrow$ $\infty)$. Then Lemma 3.1 immediately implies

$$
\liminf _{n \rightarrow \infty} Y_{n} \geq-s_{1} \text {. }
$$

Hence we obtain

$$
\limsup _{n \rightarrow \infty} \frac{X_{n+a_{n}}}{a_{n}}=\limsup _{n \rightarrow \infty}\left(\frac{1}{a_{n}} \sum_{k=n+1}^{n+a_{n}} X_{k}-Y_{n}\right) \leq s_{0}+s_{1}<\infty .
$$

Therefore

$$
\limsup _{n \rightarrow \infty} \frac{X_{n}}{n} \leq K\left(s_{0}+s_{1}\right) .
$$

Thus for every $x>K\left(s_{0}+s_{1}\right)$ we have

$$
P\left(\frac{X_{n}}{n}>x \text { i.o. }\right)=0 \text {. }
$$

Because of independence of the events $\left\{X_{n}>n x\right\}$ we obtain from the BorelCantelli lemma using the notation $Y=\frac{X}{x}$ :

$$
\begin{aligned}
\infty & >\sum_{n=1}^{\infty} P\left(X_{n}>n x\right)=\sum_{n=1}^{\infty} P(Y>n) \\
& =\sum_{k=1}^{\infty} \sum_{n=1}^{k} P(Y \in(k, k+1]) \geq \sum_{k=1}^{\infty} \int_{\{Y \in(k, k+1]\}}(Y-1) d P \\
& \geq \int_{\{Y>0\}}(Y-1) d P=E Y^{+}-P(Y>0) .
\end{aligned}
$$

Thus we have proved $\mathbf{E} Y^{+}<\infty$ and therefore $\mathbf{E} X^{+}<\infty$. $\mathbf{E} X^{-}<\infty$ can be shown similarly. From now on we assume that $\mathbf{E} X=0$. 
Now we set $Y_{n}=\frac{X_{n+1}}{a_{n}}$ and $Z_{n}=\frac{1}{a_{n}} \sum_{k=n+2}^{n+a_{n}} X_{k}$. Since $\mathbf{E} X$ exists we obtain for arbirarily small $\epsilon>0$ :

$$
P\left(Z_{n}>\epsilon\right)=P\left(\sum_{k=1}^{a_{n}-1} X_{k}>\epsilon a_{n}\right) \rightarrow 0 \quad(n \rightarrow \infty)
$$

by the weak law of large numbers. Hence Lemma 3.1 gives

$$
\limsup _{n \rightarrow \infty} Y_{n} \leq s_{0}
$$

So for every $s>s_{0}$ :

$$
P\left(X_{n}>s a_{n} \text { i.o. }\right)=0 .
$$

By the Borel-Cantelli lemma

$$
\begin{aligned}
\infty & >\sum_{n=1}^{\infty} P\left(X_{n}>s a_{n}\right)=\sum_{n=1}^{\infty} P\left(e^{g\left(\frac{X_{n}}{s}\right)}>n\right) \\
& =\sum_{k=1}^{\infty} \sum_{n=1}^{k} P\left(e^{g\left(\frac{X_{n}}{s}\right)} \in(k, k+1]\right) \\
& \geq \sum_{k=1}^{\infty}\left\{e^{g\left(\frac{X}{s}\right)} \in(k, k+1]\right\}
\end{aligned}
$$

So we have proved $\mathbf{E} e^{g\left(\frac{X}{s}\right)}<\infty$ for all $s>s_{0}$.

The second assertion $\mathbf{E} e^{g\left(\frac{X}{s}\right)}<\infty$ for all $s<-s_{1}$ follows applying the same argument to $\left(-X_{k}\right)_{k=1}^{\infty}$.

\section{Proofs of Theorems 2.1 And 2.6}

First we prove the following result which contains one part of the main theorem.

Proposition 4.1. For some $p>1$ we define $g(x)=g_{p}(x)$ and $a_{n}=(L n)^{p}$. Further let $t_{1}, t_{2}>0$ be given such that $\mathbf{E} e^{-t_{1} g(X)}<\infty$ and $\mathbf{E} e^{t_{2} g(X)}<\infty$. In particular, this implies $\mathbf{E}|X|<\infty$ and we may assume $\mathbf{E} X=0$.

Then

$$
-\frac{1}{t_{1}^{p}} \leq \liminf _{n \rightarrow \infty} \frac{1}{a_{n}} \sum_{k=n+1}^{n+a_{n}} X_{k} \leq \limsup _{n \rightarrow \infty} \frac{1}{a_{n}} \sum_{k=n+1}^{n+a_{n}} X_{k} \leq \frac{1}{t_{2}^{p}} .
$$

Proof. It suffices to prove the inequality for the upper limit. Fix $s_{2} \in\left(0, t_{2}\right)$. We decompose $X_{k}$ into $X_{k}^{\prime}=X_{k} \mathbf{1}_{\left\{X_{k} \leq s_{2}^{-p} a_{k}\right\}}$ and $X_{k}^{\prime \prime}=X_{k}-X_{k}^{\prime}$. Then

$$
\begin{aligned}
\sum_{k=1}^{\infty} P\left(X_{k}^{\prime \prime} \neq 0\right) & =\sum_{k=1}^{\infty} P\left(e^{s_{2} g(X)}>k\right) \\
& =\sum_{k=1}^{\infty} \sum_{n=k}^{\infty} P\left(e^{s_{2} g(X)} \in(n, n+1]\right) \leq \mathbf{E} e^{s_{2} g(X)}<\infty
\end{aligned}
$$


Thus $P\left(X_{k}^{\prime \prime} \neq 0\right.$ i.o. $)=0$ and therefore

$$
\lim _{n \rightarrow \infty} \frac{1}{a_{n}} \sum_{k=n+1}^{n+a_{n}} X_{k}^{\prime \prime}=0
$$

Since $1 \leq a_{n+a_{n}} / a_{n} \rightarrow 1$ as $n \rightarrow \infty$ it suffices to consider only $n$ large enough that for some $\tilde{s}_{2} \in\left(s_{2}, t_{2}\right)$ :

$$
s_{2}\left(\frac{a_{n+a_{n}}}{a_{n}}\right)^{1 / q} \leq \tilde{s}_{2}<t_{2}
$$

Now observe that $\mathbf{E} X_{k}^{\prime} \leq 0$ for all $k \in \mathbb{N}, \mathbf{E} X^{2}<\infty$ and $\mathbf{E}\left(X^{2} e^{\tilde{s}_{2} g(X)}\right)<\infty$. Then (3.2) yields for $k=n+1, \ldots, n+a_{n}$ and $t=s_{2}^{p} a_{n}^{-1 / q}$ :

$$
\begin{aligned}
\mathbf{E} e^{t X_{k}^{\prime}} & \leq 1+\frac{t^{2}}{2} \mathbf{E}\left(\left(X^{\prime}\right)_{k}^{2} H\left(t X_{k}^{\prime}\right)\right) \\
& \leq 1+\frac{t^{2}}{2}\left(\mathbf{E} X^{2}+\mathbf{E}\left(\left(X^{\prime}\right)_{k}^{2} e^{t X_{k}^{\prime}} \mathbf{1}_{\left\{X_{k}^{\prime} \geq 0\right\}}\right)\right) \\
& \leq 1+\frac{t^{2}}{2}\left(\mathbf{E} X^{2}+\mathbf{E}\left(X^{2} \exp \left\{t s_{2}^{-p / q} a_{k}^{1 / q} g(X)\right\}\right)\right) \\
& \leq 1+\frac{s_{2}^{2 p}}{2 a_{n}^{2 / q}}\left(\mathbf{E} X^{2}+\mathbf{E}\left(X^{2} e^{\tilde{s}_{2} g(X)}\right)\right) \leq \exp \left\{O\left(a_{n}^{-2 / q}\right)\right\}
\end{aligned}
$$

where we have used (3.3) in the final step. Hence we obtain for any $x>0$ :

$$
\begin{aligned}
P\left(\sum_{k=n+1}^{n+a_{n}} X_{k}^{\prime}>x a_{n}\right) & \leq e^{-t x a_{n}} \prod_{k=n+1}^{n+a_{n}} \mathbf{E} e^{t X_{k}^{\prime}} \\
& \leq \exp \left\{-x s_{2}^{p} \log n+O\left(a_{n}^{1-2 / q}\right)\right\} \\
& \leq \exp \left\{-x s_{2}^{p} \log n+o(\log n)\right\}
\end{aligned}
$$

which yields a convergent series if $x>s_{2}^{-p}$. Since $s_{2} \in\left(0, t_{2}\right)$ was arbitrary this proves

$$
\limsup _{n \rightarrow \infty} \frac{1}{a_{n}} \sum_{k=n+1}^{n+a_{n}} X_{k}^{\prime} \leq \frac{1}{t_{2}^{p}} .
$$

Now the assertion follows from (4.2) and (4.3).

The same proof yields the following variant of Proposition 4.1:

Proposition 4.2. For some $p>1$ we define $g(x)=g_{p}(x)$ and $a_{n}=(L n)^{p}$. Let $\gamma \geq 1$.

Let $t_{1}, t_{2}>0$ be given such that $\mathbf{E} e^{-t_{1} g(X)}<\infty$ and $\mathbf{E} e^{t_{2} g(X)}<\infty$. In particular, this implies $\mathbf{E}|X|<\infty$ and we may assume $\mathbf{E} X=0$.

Then

$$
-\frac{1}{\gamma t_{1}^{p}} \leq \liminf _{n \rightarrow \infty} \frac{1}{a_{n^{\gamma}}} \sum_{k=\left[n^{\gamma}\right]+1}^{\left[n^{\gamma}\right]+a_{n} \gamma} X_{k} \leq \limsup _{n \rightarrow \infty} \frac{1}{a_{n^{\gamma}}} \sum_{k=\left[n^{\gamma}\right]+1}^{\left[n^{\gamma}\right]+a_{n} \gamma} X_{k} \leq \frac{1}{\gamma t_{2}^{p}} .
$$


Proof. (Theorem 2.1) We first assume that (i) holds. Then the expected value $\mu=\mathbf{E} X$ exists. Now $\mathbf{E} e^{t g(X-\mu)}<\infty$ for $t \in\left(-t_{1}, t_{2}\right)$ and $\mathbf{E} e^{t g(X-\mu)}=\infty$ for $t \notin\left[-t_{1}, t_{2}\right]$ by Lemma 3.5. Therefore we may without loss of generality assume that $\mu=0$ since otherwise $X_{k}$ may be replaced by $X_{k}-\mu$.

Proposition 4.1 immediately yields

$$
\limsup _{n \rightarrow \infty} \frac{1}{a_{n}} \sum_{k=n+1}^{n+a_{n}} X_{k} \leq \frac{1}{t_{2}^{p}} \text { a.s. }
$$

Similarly we can prove the corresponding statement for the lower limit. On the other hand we know from Kolmogorov's 0-1 law that the upper and lower limit are constant almost surely. If now

$$
\limsup _{n \rightarrow \infty} \frac{1}{a_{n}} \sum_{k=n+1}^{n+a_{n}} X_{k} \leq \frac{1}{t^{p}} \text { a.s. }
$$

with some $t>t_{2}$ then we obtain from Proposition 3.6 that $\mathbf{E} e^{g(s X)}=$ $\mathbf{E} e^{s^{1 / p} g(X)}<\infty$ for all $s \in\left(0, t^{p}\right)$, thus particularly for an $s$ with $s^{1 / p}>t_{2}$ contradicting the assumptions. So we have proved

$$
\limsup _{n \rightarrow \infty} \frac{1}{a_{n}} \sum_{k=n+1}^{n+a_{n}} X_{k}=\frac{1}{t_{2}^{p}} \text { a.s. }
$$

and the proof of

$$
\liminf _{n \rightarrow \infty} \frac{1}{a_{n}} \sum_{k=n+1}^{n+a_{n}} X_{k}=-\frac{1}{t_{1}^{p}} \text { a.s. }
$$

is similar.

Now we assume (ii). By Proposition 3.6 $\mathrm{E}^{\operatorname{tg}(X)}<\infty$ for all $t$ in a neighborhood of 0 . So $\mathbf{E}|X|<\infty$ and we denote $\mathbf{E} X=\mu$. If we replace $X_{k}$ by $X_{k}-\mu$ again, if necessary, we may assume without loss of generality that $\mu=0$.

Then Proposition 3.6 implies $\mathbf{E} e^{\operatorname{tg}(X)}<\infty$ for all $t \in\left(-t_{1}, t_{2}\right)$. If $\mathbf{E} e^{\operatorname{tg}(X)}$ $<\infty$ for a $t>t_{2}$ then as in the first part of the proof

$$
\limsup _{n \rightarrow \infty} \frac{1}{a_{n}} \sum_{k=n+1}^{n+a_{n}} X_{k} \leq \frac{1}{t^{p}} \text { a.s. }
$$

would follow from Proposition 4.1 and this would contradict the assumptions. The assumption $\mathbf{E} e^{t g(X)}<\infty$ for some $t<-t_{1}$ similarly leads to a contradiction.

REMARK 4.3. In the special case where an asymptotic for the tail of the distribution function of $X$ is known Theorem 2.1 can also be proved using a large deviations result implicit in Nagaev (1979) (this can also be found with a short proof in Gantert (1996)).

Proof. (Theorem 2.6) That (i) follows from (ii) is obvious from Theorem 2.1 . 
So we assume (i). Using Proposition 4.2 we can show exactly as in the proof of Theorem 2.1 that the moment condition (i) is equivalent to

$$
\liminf _{n \rightarrow \infty} \frac{1}{a_{n^{\gamma}}} \sum_{k=\left[n^{\gamma}\right]+1}^{\left[n^{\gamma}\right]+a_{n} \gamma} X_{k}=\mu-\frac{1}{\gamma t_{1}^{p}} \text { a.s. }
$$

and

$$
\limsup _{n \rightarrow \infty} \frac{1}{a_{n^{\gamma}}} \sum_{k=\left[n^{\gamma}\right]+1}^{\left[n^{\gamma}\right]+a_{n} \gamma} X_{k}=\mu+\frac{1}{\gamma t_{2}^{p}} \text { a.s. }
$$

Since $\gamma \geq 1$ is arbitrary we find for every $s \in\left[\mu-t_{1}^{-p}, \mu+t_{2}^{-p}\right]$ a subsequence with an almost sure upper or lower limit that equals $s$. The remainder follows from a standard argument: If we choose a countable dense subset $S$ of $\left[\mu-t_{1}^{-p}, \mu+t_{2}^{-p}\right]$ it follows by excluding the exeptional sets corresponding to elements of $S$ :

$$
\mathbf{C}\left(\left\{\frac{1}{a_{n}} \sum_{k=n+1}^{n+a_{n}} X_{k}\right\}\right) \supseteq S \text { a.s. }
$$

Since the set of limit points must be closed we obtain

$$
\mathbf{C}\left(\left\{\frac{1}{a_{n}} \sum_{k=n+1}^{n+a_{n}} X_{k}\right\}\right) \supseteq\left[\mu-\frac{1}{t_{1}^{p}}, \mu+\frac{1}{t_{2}^{p}}\right] \text { a.s. }
$$

The reverse inclusion

$$
\mathbf{C}\left(\left\{\frac{1}{a_{n}} \sum_{k=n+1}^{n+a_{n}} X_{k}\right\}\right) \subseteq\left[\mu-\frac{1}{t_{1}^{p}}, \mu+\frac{1}{t_{2}^{p}}\right] \text { a.s. }
$$

is an obvious consequence of Theorem 2.1. This completes the proof.

\section{Proofs of Theorems 2.7 and 2.9}

Proposition 5.1. For some $p>1$ we define $g(x)=g_{p}(x)$ and $a_{n}=L^{p} n$. Further let $t_{1}, t_{2}>0$ be given.

We assume $\mathbf{E} e^{t g(X)}<\infty$ for all $t \in\left[-t_{1}, t_{2}\right]$ and $\mathbf{E} X=0$.

Finally let $\left(u_{n}\right)_{n=1}^{\infty}$ and $\left(l_{n}\right)_{n=1}^{\infty}$ be monotonically decreasing sequences of reals with $0 \leq l_{n}, u_{n} \leq 1, a_{n} u_{n} \rightarrow \infty, a_{n} l_{n} \rightarrow \infty \quad(n \rightarrow \infty)$ and $u=\lim _{n \rightarrow \infty} u_{n}$ and $l=\lim _{n \rightarrow \infty} l_{n}$. Set

$$
U_{n}=\sum_{k=n+1}^{n+a_{n}} X_{k} \mathbf{1}_{\left\{-\frac{l_{n}}{t_{1}^{p}} a_{n} \leq X_{k} \leq \frac{u_{n}}{t_{2}^{p}} a_{n}\right\}} .
$$

Then

$$
-\frac{l^{1 / q}}{t_{1}^{p}} \leq \liminf _{n \rightarrow \infty} \frac{1}{a_{n}} U_{n} \leq \limsup _{n \rightarrow \infty} \frac{1}{a_{n}} U_{n} \leq \frac{u^{1 / q}}{t_{2}^{p}} .
$$

Proof. Set $Y_{k}^{(n)}=X_{k} \mathbf{1}_{\left\{-l_{n} t_{1}^{-p} a_{n} \leq X_{k} \leq u_{n} t_{2}^{-p} a_{n}\right\}}$ and $\mu_{n}=\mathbf{E} Y_{k}^{(n)}$. Observe that $\mu_{n} \rightarrow 0$ as $n \rightarrow \infty$. Choose $\bar{\delta}>0$ and $s_{2} \in\left(0, t_{2}\right)$ arbitrarily but 
fixed. We now follow the lines of the proof of Proposition 4.1. Choosing $t=s_{2}^{p}\left(u_{n}+\delta\right)^{-1 / q} a_{n}^{-1 / q}$ we obtain

$$
\begin{aligned}
\mathbf{E} e^{t Y_{k}^{(n)}} & \leq 1+t \mu_{n}+\frac{t^{2}}{2} \mathbf{E}\left(Y_{k}^{(n)} H\left(t Y_{k}^{(n)}\right)\right) \\
& \leq \exp \left\{o\left(a_{n}^{-1 / q}\right)+O\left(a_{n}^{-2 / q}\right)\right\}=\exp \left\{o\left(a_{n}^{-1 / q}\right)\right\} .
\end{aligned}
$$

Thus for $x>0$ :

$P\left(U_{n}>x a_{n}\right) \leq e^{-t x a_{n}} \prod_{k=n+1}^{n+a_{n}} \mathbf{E} e^{t Y_{k}^{(n)}} \leq \exp \left\{-\frac{x s_{2}^{p}}{\left(u_{n}+\delta\right)^{1 / q}} \log n+o(\log n)\right\}$

which yields a convergent series if $x>(u+\delta)^{1 / q} s_{2}^{-p}$.

Hence $\limsup \sup _{n \rightarrow \infty} U_{n} / a_{n} \leq(u+\delta)^{1 / q} s_{2}^{-p}$ a.s. and, since $\delta>0$ and $s_{2} \in$ $\left(0, t_{2}\right)$ where arbitrary,

$$
\limsup _{n \rightarrow \infty} \frac{1}{a_{n}} U_{n} \leq \frac{u^{1 / q}}{t_{2}^{p}} \text { a.s. }
$$

Thus the upper inequality of the assertion follows and the lower inequality is proved similarly.

Before we can prove the relevant theorems we need some information about how often very large terms may occur.

Proposition 5.2. For some $p>1$ we define $g(x)=g_{p}(x)$ and $a_{n}=(L n)^{p}$. Further let $t_{1}, t_{2}>0$ be given.

We assume $\mathbf{E} e^{t g(X)}<\infty$ for all $t \in\left[-t_{1}, t_{2}\right]$.

Finally let $\left(r_{n}\right)_{n=1}^{\infty}$ and $\left(k_{n}\right)_{n=1}^{\infty}$ be monotonically increasing sequences of positive numbers satisfying $k_{n} \rightarrow \infty$ and $k_{n} r_{n}^{-p} \rightarrow 0$ for $n \rightarrow \infty$ and also

$$
\liminf _{n \rightarrow \infty} \frac{a_{n}}{k_{n} L^{\tau} n}>0
$$

for some $\tau>0$.

Then

$$
\begin{aligned}
\lim _{n \rightarrow \infty} \frac{1}{r_{n}} \sum_{k=n+1}^{n+a_{n}} \mathbf{1}_{\left\{X_{k}>\frac{a_{n}}{k_{n}}\right\}} & =\lim _{n \rightarrow \infty} \frac{1}{r_{n}} \sum_{k=n+1}^{n+a_{n}} \mathbf{1}_{\left\{X_{k}<-\frac{a_{n}}{k_{n}}\right\}} \\
& =\lim _{n \rightarrow \infty} \frac{1}{r_{n}} \sum_{k=n+1}^{n+a_{n}} \mathbf{1}_{\left\{\left|X_{k}\right|>\frac{a_{n}}{k_{n}}\right\}}=0 \text { a.s. }
\end{aligned}
$$

Proof. Set

$$
J_{n}=\sum_{k=n+1}^{n+a_{n}} 1_{\left\{X_{k}>\frac{a_{n}}{k_{n}}\right\}}
$$


We may assume without loss of generality that $k_{n} r_{n}^{-p} \leq 1$ for all $n \in \mathbb{N}$.

Choose an arbitrary but fixed $x>0$. Then we have for any $t>0$ :

$$
\begin{aligned}
P\left(J_{n}>x r_{n}\right) & =P\left(e^{t J_{n}-t x r_{n}}>1\right) \leq e^{-t x r_{n}} \mathbf{E} e^{t J_{n}} \\
& =e^{-t x r_{n}}\left(e^{t} P\left(X>\frac{a_{n}}{k_{n}}\right)+P\left(X \leq \frac{a_{n}}{k_{n}}\right)\right)^{\left[a_{n}\right]} \\
& \leq e^{-t x r_{n}}\left(1+e^{t} P\left(e^{t_{2} g(X)}>e^{t_{2} g\left(a_{n} / k_{n}\right)}\right)\right)^{\left[a_{n}\right]} \\
& \leq e^{-t x r_{n}}\left(1+e^{t} \frac{\mathbf{E} e^{t_{2} g(X)}}{e^{t_{2} L n / k_{n}^{1 / p}}}\right)^{\left[a_{n}\right]} .
\end{aligned}
$$

In the sequel we use the notation $M\left(t_{2}\right)=\mathbf{E} e^{t_{2} g(X)}$. Choose $t_{0} \in\left(0, t_{2}\right)$ and set $t=t_{0} L n k_{n}^{-1 / p}$. By (3.3) we obtain for all $n$ such that $t_{0} x r_{n} k_{n}^{-1 / p} \geq 2$ :

$$
\begin{aligned}
P\left(J_{n}>x r_{n}\right) & \leq \exp \left\{-t_{0} x \operatorname{Ln} \frac{r_{n}}{k_{n}^{1 / p}}\right\}\left(1+\frac{M\left(t_{2}\right)}{\exp \left\{\left(t_{2}-t_{0}\right) L n / k_{n}^{1 / p}\right\}}\right)^{\left[a_{n}\right]} \\
& \leq \exp \left\{-2 L n+M\left(t_{2}\right) \frac{\left[a_{n}\right]}{\exp \left\{\left(t_{2}-t_{0}\right) L n / k_{n}^{1 / p}\right\}}\right\} .
\end{aligned}
$$

Since there exists a $\tau>0$ by assumption such that

$$
\frac{L n}{k_{n}^{1 / p}} \geq \xi L^{\tau} n
$$

for some $\xi>0$ and sufficiently large $n$ it follows that

$$
\frac{\left[a_{n}\right]}{\exp \left\{\left(t_{2}-t_{0}\right) L n / k_{n}^{1 / p}\right\}} \rightarrow 0 \quad(n \rightarrow \infty) .
$$

Hence there exists a $K>0$ with

$$
P\left(J_{n}>x r_{n}\right) \leq K n^{-2} .
$$

This yields a convergent series and therefore

$$
\limsup _{n \rightarrow \infty} \frac{1}{r_{n}} J_{n} \leq x
$$

Since $J_{n} \geq 0$ for all $n$ and $x>0$ was arbitrary this completes the proof. The other assertions can be proved similarly.

Proof. (Theorem 2.7) Note that condition (2.1) implies $\alpha<p$. Set $k_{n}=r_{n}^{\alpha}$. Then obviously $\liminf _{n \rightarrow \infty} \frac{a_{n}}{k_{n}(L n)^{\tau}}>0$ for some $\tau>0$ and also

$$
\frac{k_{n}}{r_{n}}=r_{n}^{\alpha-1} \rightarrow \infty \quad(n \rightarrow \infty) \text { and } \frac{k_{n}}{r_{n}^{p}}=r_{n}^{\alpha-p} \rightarrow 0 \quad(n \rightarrow \infty) .
$$

Now define

$$
U_{n}=\sum_{k=n+1}^{n+a_{n}} X_{k} \mathbf{1}_{\left\{\left|X_{k}\right| \leq \frac{a_{n}}{k_{n}}\right\}} \text { and } J_{n}=\sum_{k=n+1}^{n+a_{n}} \mathbf{1}_{\left\{\left|X_{k}\right|>\frac{a_{n}}{k_{n}}\right\}} .
$$


Then $\lim _{n \rightarrow \infty} J_{n} / r_{n}=0$ according to Proposition 5.2. Hence for almost all $\omega \in \Omega$ there exists an $N(\omega)$ such that for all $n \geq N(\omega)$ :

$$
0 \leq J_{n} \leq \frac{1}{2} r_{n},
$$

i.e. for $n \geq N$ at most $r_{n} / 2$ summands of a modulus larger larger than $a_{n} / k_{n}$ occur. This means we have eliminated all summands of modulus larger than $a_{n} / k_{n}$ from the sum $\sum_{k=n+1}^{n+a_{n}} X_{k}$ in case $n \geq N$. Therefore $U_{n}$ and $\sum_{k=n+1}^{n+a_{n}} X_{k}$ differ by at most $r_{n}$ summands of modulus $\leq a_{n} / k_{n}$. For fixed $\omega$ and $n \geq N(\omega)$ this yields

$$
\frac{1}{a_{n}}\left|U_{n}-\sum_{k=n+1}^{n+a_{n}} X_{k}\right| \leq \frac{r_{n}}{a_{n}} \cdot \frac{a_{n}}{k_{n}}=\frac{r_{n}}{k_{n}} \rightarrow 0 \quad(n \rightarrow \infty),
$$

hence

$$
\frac{1}{a_{n}}\left(U_{n}-\sum_{k=n+1}^{n+a_{n}} X_{k}\right) \rightarrow 0 \text { a.s. } \quad(n \rightarrow \infty)
$$

Since Proposition 5.1 implies $\frac{1}{a_{n}} U_{n} \rightarrow \mu \quad(n \rightarrow \infty)$ the assertion follows.

Thus we have found out that a strong law holds if we exclude a sequence of extremal terms from the sums which is increasing to infinity but not growing too fast. In the sequel we prove that this result is best possible in the sense that it is not sufficient to exclude only finitely many terms. Since we have only shown an inequality for the upper and lower limits of truncated random variables in Proposition 5.1 we cannot compare the sequence with the finitely many extremal terms to the sequence of truncated variables but have to proceed in a different way.

Lemma 5.3. For some $p>1$ we define $g(x)=g_{p}(x)$ and $a_{n}=(L n)^{p}$. Further let $t_{0}, t_{1}, t_{2}>0$ be given.

We assume $\mathbf{E} e^{t g(X)}<\infty$ for all $t \in\left[-t_{1}, t_{0}\right]$ and $\mathbf{E} e^{t_{2} g(X)}=\infty$.

Further let $\epsilon>0$ and $r \in \mathbb{N}$ with $\epsilon<\frac{1}{r t_{2}}$. Finally let $\left(l_{n}\right)_{n=1}^{\infty}$ be a monotonically decreasing sequence with $0 \leq l_{n} \leq 1, \lim _{n \rightarrow \infty} l_{n}=l$ with $L n l_{n}>L^{\tau} n$ for all sufficiently large $n$ with some fixed $\tau>0$.

Then for the sequence of sets

$$
\begin{aligned}
B_{n}= & \left\{X_{k}>\epsilon^{p} a_{n} \text { for at least } r \text { indices } k=n+1, \ldots, n+\left[a_{n}\right],\right. \\
& \left.X_{k} \geq \frac{-l_{n}^{p} a_{n}}{t_{1}^{p}} \text { for all } k=n+1, \ldots, n+\left[a_{n}\right]\right\},
\end{aligned}
$$

it follows that

$$
P\left(B_{n} \text { i.o. }\right)=1 \text {. }
$$


Proof. Set

$$
A_{n}=\left\{X_{k}>\epsilon^{p} a_{n} \text { for at least } r \text { indices } k=n+1, \ldots, n+\left[a_{n}\right]\right\}
$$

and

$$
C_{n}=\left\{X_{k} \geq-\frac{l_{n}^{p} a_{n}}{t_{1}^{p}} \text { for all } k=n+1, \ldots, n+\left[a_{n}\right]\right\} .
$$

Further set $k_{n}=\left[L\left(a_{n}\right)\right]$. Then

$$
\begin{aligned}
& P\left(B_{n}\right)=P\left(A_{n} \cap C_{n}\right) \\
& =\sum_{\nu=r}^{a_{n}} P\left(X_{i}>\epsilon^{p} a_{n} \text { for exactly } \nu \text { indices } i=n+1, \ldots, n+\left[a_{n}\right]\right. \text {; } \\
& \left.X_{i} \geq-l_{n}^{p} a_{n} / t_{1}^{p} \text { for all } i=n+1, \ldots, n+\left[a_{n}\right]\right) \\
& \geq \sum_{\nu=r}^{k_{n}}\left(\begin{array}{c}
{\left[a_{n}\right]} \\
\nu
\end{array}\right) P\left(X_{1}>\epsilon^{p} a_{n}, \ldots, X_{\nu}>\epsilon^{p} a_{n},\right. \\
& X_{\nu+1} \in\left(-l_{n}^{p} a_{n} / t_{1}^{p}, \epsilon^{p} a_{n}\right], \ldots, \\
& \left.X_{a_{n}} \in\left(-l_{n}^{p} a_{n} / t_{1}^{p}, \epsilon^{p} a_{n}\right]\right) \\
& \geq \sum_{\nu=r}^{k_{n}}\left(\begin{array}{c}
{\left[a_{n}\right]} \\
\nu
\end{array}\right) P\left(X>\epsilon^{p} a_{n}\right)^{\nu} \\
& \cdot P\left(X \leq \epsilon^{p} a_{n}\right)^{\left[a_{n}\right]-\nu} \frac{P\left(X \in\left(-l_{n}^{p} a_{n} / t_{1}^{p}, \epsilon^{p} a_{n}\right]\right)^{\left[a_{n}\right]}}{P\left(X \leq \epsilon^{p} a_{n}\right)^{\left[a_{n}\right]-\left[k_{n}\right]}} .
\end{aligned}
$$

Now Lemma 3.2 implies

$$
\begin{aligned}
& P\left(X \in\left(\frac{-l_{n}^{p} a_{n}}{t_{1}^{p}}, \epsilon^{p} a_{n}\right]\right)^{\left[a_{n}\right]} \\
\sim & \exp \left\{\left[a_{n}\right]\left(P\left(X \leq-\frac{l_{n}^{p} a_{n}}{t_{1}^{p}}\right)+P\left(X>\epsilon^{p} a_{n}\right)\right)\right\} \sim 1
\end{aligned}
$$

as $n \rightarrow \infty$ and

$$
P\left(X \leq \epsilon^{p} a_{n}\right)^{\left[a_{n}\right]-\left[k_{n}\right]} \sim \exp \left\{\left(\left[a_{n}\right]-\left[k_{n}\right]\right) P\left(X>\epsilon^{p} a_{n}\right)\right\} \sim 1 \quad(n \rightarrow \infty) .
$$

Therefore we have for sufficiently large $n$ :

$$
\begin{aligned}
P\left(B_{n}\right) & \geq \frac{1}{2} \sum_{\nu=r}^{k_{n}}\left(\begin{array}{c}
{\left[a_{n}\right]} \\
\nu
\end{array}\right) P\left(X>\epsilon^{p} a_{n}\right)^{\nu} \cdot P\left(X \leq \epsilon^{p} a_{n}\right)^{\left[a_{n}\right]-\nu} \\
& \geq \frac{1}{2} P\left(A_{n}\right)-\frac{1}{2} P\left(X_{i}>\epsilon^{p} a_{n}\right.
\end{aligned}
$$

for at least $k_{n}$ indices $\left.k=1, \ldots,\left[a_{n}\right]\right)$.

Choose $\gamma>1$ small enough that $\gamma^{4} \epsilon<1 /\left(r t_{2}\right)$. We want to show that

$$
\sum_{n=1}^{\infty} P\left(B_{n \gamma}\right)=\infty
$$


Since $\frac{L^{2}\left(a_{n}\right)}{a_{n}} \rightarrow 0 \quad(n \rightarrow \infty)$ Lemma 3.3 yields:

$$
\begin{aligned}
& P\left(X_{i}>\epsilon^{p} a_{n} \text { for at least } k_{n} \text { indices } k=1, \ldots,\left[a_{n}\right]\right) \\
\sim & \frac{a_{n}^{k_{n}}}{k_{n} !} P\left(X>\epsilon^{p} a_{n}\right)^{k_{n}} \leq e^{\left(L a_{n}-t_{0} \epsilon L n\right) k_{n}}\left(\mathbf{E} e^{t_{0} g(X)}\right)^{k_{n}} \\
\leq & \exp \left\{-L n k_{n} t_{0} \epsilon+o\left(L n k_{n}\right)\right\} .
\end{aligned}
$$

Therefore

$$
\sum_{n=1}^{\infty} P\left(X_{i}>\epsilon^{p} a_{n} \text { for at least } k_{n} \text { indices } k=1, \ldots,\left[a_{n}\right]\right)<\infty .
$$

Hence it suffices to prove

$$
\sum_{n=1}^{\infty} P\left(A_{n^{\gamma}}\right)=\infty
$$

Lemma 3.3 yields

$$
P\left(A_{n}\right) \sim \frac{a_{n}^{r} P\left(X>\epsilon^{p} a_{n}\right)^{r}}{r !} \quad(n \rightarrow \infty) .
$$

Thus we must show:

$$
\sum_{n=1}^{\infty} a_{n^{\gamma}}^{r} P\left(X>\epsilon^{p} a_{n^{\gamma}}\right)^{r}=\infty .
$$

To prove this by contradiction, we assume that

$$
\sum_{n=1}^{\infty} a_{n^{\gamma}}^{r} P\left(X>\epsilon^{p} a_{n^{\gamma}}\right)^{r}<\infty
$$

By the substitution $u=\epsilon^{p} \gamma^{2 p} \log ^{p} y$, i.e. $y=e^{u^{1 / p} /\left(\epsilon \gamma^{2}\right)}$, we can conclude

$$
\begin{aligned}
& \infty>\sum_{n=1}^{\infty} a_{n^{\gamma}}^{r} P\left(X>\epsilon^{p} a_{n^{\gamma}}\right)^{r} \\
& \geq C \sum_{n=1}^{\infty} \int_{n}^{n+1} \log ^{p r}(n) P\left(X>\epsilon^{p} \log ^{p}\left(n^{\gamma}\right)\right)^{r} d y \\
& \geq C \sum_{n=1}^{\infty} \int_{n}^{n+1} \log ^{p r}(y) P\left(X>(\gamma \epsilon)^{p} \log ^{p} y\right)^{r} d y \\
&=C \int_{0}^{\infty} u^{r} P\left(X>u / \gamma^{p}\right)^{r} d \epsilon^{u^{1 / p} /\left(\epsilon \gamma^{2}\right)} \\
& \geq C \sum_{n=1}^{\infty} \int_{n}^{n+1} u^{r-1+1 / p} P\left(X>u / \gamma^{p}\right)^{r} e^{u^{1 / p} /\left(\epsilon \gamma^{2}\right)} d u \\
& \geq C \sum_{n=1}^{\infty} \int_{n}^{n+1} n^{r-1+1 / p} P(X>n)^{r} e^{n^{1 / p} /\left(\epsilon \gamma^{2}\right)} d u \\
& \geq C \sum_{n=1}^{\infty} P(X>n)^{r} e^{n^{1 / p} /\left(\epsilon \gamma^{2}\right)} .
\end{aligned}
$$


Hence

$$
P(X>n)^{r} e^{n^{1 / p} /\left(\epsilon \gamma^{2}\right)} \leq 1 \text { i.e. } P(X>n) \leq e^{-n^{1 / p} /\left(r \epsilon \gamma^{2}\right)}
$$

for large $n$. This implies for sufficiently large $n$ :

$$
P(g(X)>n)=P\left(X>n^{p}\right) \leq \exp \left\{\frac{-\left[n^{p}\right]^{1 / p}}{r \epsilon \gamma^{2}}\right\} \leq \exp \left\{\frac{-n}{r \epsilon \gamma^{3}}\right\} .
$$

For large $x, x \in[n, n+1]$, we obtain

$$
P(g(X)>x) \leq e^{-n /\left(r \epsilon \gamma^{3}\right)} \leq e^{-x /\left(r \epsilon \gamma^{4}\right)} .
$$

For $t \in\left(0, \frac{1}{r \in \gamma^{4}}\right)$ we get

$$
\mathbf{E} e^{t g(X)}=\int_{-\infty}^{\infty} e^{t u} P(g(X)>u) d u<\infty
$$

Since $t_{2}<\frac{1}{r \epsilon \gamma^{4}}$ this particularly holds for $t=t_{2}$ and this contradicts the assumptions.

Hence $\sum_{n=1}^{\infty} P\left(B_{n} \gamma\right)=\infty$. For sufficiently large $n$ we moreover know that $[(n+$ $\left.1)^{\gamma}\right]>\left[n^{\gamma}\right]+\left[a_{n} \gamma\right]+1$ which means that $B_{(n+1)^{\gamma}}$ and $B_{n^{\gamma}}$ are independent. The Borel-Cantelli lemma implies $P\left(B_{n}\right.$ i.o. $)=1$.

Proof. (Theorem 2.9) Let $l_{n} \searrow 0$ be according to the assumptions of Lemma 5.3, e.g. $l_{n}=(\log n)^{-1 / 2}$. Then Proposition 5.1 implies

$$
\frac{1}{a_{n}} \sum_{k=n+1}^{n+a_{n}} X_{k} \mathbf{1}_{\left\{-l_{n} a_{n} / t_{1}^{p} \leq X_{k} \leq l_{n} a_{n} / t_{0}^{p}\right\}} \rightarrow 0 \text { a.s. } \quad(n \rightarrow \infty) .
$$

Choose an $\epsilon<1 /\left(r t_{2}\right)$ and an $\omega \in \limsup _{n \rightarrow \infty} B_{n}$ where the sets $B_{n}$ be defined as in Lemma 5.3. Then there exists a sequence $\left(n_{\nu}\right)_{\nu=1}^{\infty}, n_{\nu} \in \mathbb{N}$, such that $\omega \in B_{n_{\nu}}$ for all $\nu \in \mathbb{N}$. For such a fixed $\omega$ we have:

$$
\begin{aligned}
\limsup _{n \rightarrow \infty} \frac{1}{a_{n}} \sum_{k=n+1}^{n+a_{n}} X_{k} & \geq \limsup _{\nu \rightarrow \infty} \frac{1}{a_{n}} \sum_{k=n_{\nu}+1}^{n_{\nu}+a_{n_{\nu}}} X_{k} \\
& \geq \epsilon^{p}-\left|\limsup _{n \rightarrow \infty} \frac{1}{a_{n}} \sum_{k=n+1}^{n+a_{n}} X_{k} \mathbf{1}_{\left\{-l_{n} a_{n} / t_{1}^{p} \leq X_{k} \leq l_{n} a_{n} / t_{0}^{p}\right\}}\right| \\
& \geq \epsilon^{p} .
\end{aligned}
$$

Since $\epsilon<1 /\left(r t_{2}\right)$ was arbitrary this completes the proof.

\section{Concluding REMARKS}

Now we briefly state two results that show that Theorem 2.1 does not depend on the special form of the moment condition $\mathbf{E} e^{t \operatorname{sgn} X|X|^{1 / p}}$. Since the proofs for all preliminary results and the theorems themselves are very similar to the case of Theorem 2.1 we omit them. For the notion of regular variation used in the sequel we refer the reader to the monograph by Bingham, Goldie and Teugels (1987). 
THEOREM 6.1. Let $p>1$ and $g: \mathbb{R} \rightarrow \mathbb{R}$ be a function with the following properties:

- $g$ is strictly increasing.

- $g$ is regularly varying of degree $\frac{1}{p}$.

Again we define $a_{n}=g^{-1}(\mathrm{Ln})$. Finally let real numbers $t_{1}, t_{2} \in(0, \infty]$ be given.

Then the following are equivalent:

(i) $\mathbf{E} e^{t g(X)}<\infty$ for $t \in\left(-t_{1}, t_{2}\right), \mathbf{E} e^{\operatorname{tg}(X)}=\infty$ for $t \notin\left[-t_{1}, t_{2}\right]$ and $\mathbf{E} X=$ $\mu$.

(ii) $\liminf _{n \rightarrow \infty} \frac{1}{a_{n}} \sum_{k=n+1}^{n+a_{n}} X_{k}=\mu-\frac{1}{t_{1}^{p}}$ a.s. and

$$
\limsup _{n \rightarrow \infty} \frac{1}{a_{n}} \sum_{k=n+1}^{n+a_{n}} X_{k}=\mu+\frac{1}{t_{2}^{p}} \text { a.s. }
$$

Theorem 6.2. Let $\nu \in \mathbb{N}$ and Let $\nu \in \mathbb{N}$ and $g(x)=x / L_{\nu}|x|$ for all $x \in \mathbb{R}$ as well as $a_{n}=g^{-1}(L n)$.

Further let $t_{1}, t_{2} \in(0, \infty]$.

Then the following are equivalent:

(i) $\mathbf{E} e^{\operatorname{tg}(X)}<\infty$ for $t \in\left(-t_{1}, t_{2}\right), \mathbf{E} e^{\operatorname{tg}(X)}=\infty$ for $t \notin\left[-t_{1}, t_{2}\right]$ and $\mathbf{E} X=$ $\mu$.

(ii) $\liminf _{n \rightarrow \infty} \frac{1}{a_{n}} \sum_{k=n+1}^{n+a_{n}} X_{k}=\mu-\frac{1}{t_{1}}$ a.s. and

$$
\limsup _{n \rightarrow \infty} \frac{1}{a_{n}} \sum_{k=n+1}^{n+a_{n}} X_{k}=\mu+\frac{1}{t_{2}} \text { a.s. }
$$

REMARK 6.3. Condition (ii) can again be sharpened to

$$
\mathbf{C}\left(\left\{\frac{1}{a_{n}} \sum_{k=n+1}^{n+a_{n}} X_{k}\right\}\right)=\left[\mu-\frac{1}{t_{1}}, \mu+\frac{1}{t_{2}}\right] \text { a.s. }
$$

REMARK 6.4. The case $t_{1}=t_{2}=\infty$ again gives a strong law in the classical sense.

- According to a special case of the well-known Komlós-Major-Tusnády approximation (see Komlós, Major and Tusnády $(1975,1976)$ and Major (1976)) a sequence of independent identically distributed random variables satisfying $\mathbf{E} \phi(|X|)<\infty$ can be approximated almost surely by a Wiener process on a suitable probability space with the almost sure error being $O\left(\phi^{-1}(n)\right)$ for a large class of functions $\phi:[0, \infty) \rightarrow$ $[0, \infty)$.

Now one can raise the question as to whether the $O(\cdot)$ occuring in the error term can be replaced by $o(\cdot)$. It has been known for a long time that in the case $\phi(x)=x^{p}$ with some $p>2$ the answer is positive while it is negative for $\phi(x)=e^{t x}$ with some $t>0$. The latter essentially is a consequence of the Erdös-Rényi law. Shao (1989) proved that in the case $\phi(x)=e^{t x^{1 / p}}$ with $p>1 O(\cdot)$ cannot be replaced by $o(\cdot)$ in general either. Shao's result also is an immediate consequence of Theorem 2.1 because a strong approximation with error term $o\left(\log ^{p} n\right)$ would imply 
a strong law in the sense of almost sure convergence to the mean in the situation of Theorem 2.1. But we just have proved that a strong law of this kind does not hold so a strong approximation which is sufficient for it cannot hold either.

The question as to whether a strong approximation with error term $o\left(\log ^{p} n\right)$ holds under the stronger condition $\mathbf{E} e^{t|X|^{1 / p}}<\infty$ for all $t>0$ which would still be possible according to the case $t_{1}=t_{2}=\infty$ of Theorem 2.1 remains open.

- As already mentioned in the introduction one motivation for dealing with moving averages of random variables is the fact that they often imply strong laws for other summability methods when some appropriate analytical theorems are applied. Using our strong laws and results of Bingham and Goldie (1988) or Stadtmüller (1995) strong laws for a variety of summability methods can easily be obtained.

\section{REFERENCES}

DE AcostA, A. and KuelBs, J. (1983). Limit theorems for moving averages of independent random vectors. Z. Wahrscheinlichkeitstheorie verw. Geb. 64, 67-123.

Bingham, N.H. (1985). On Tauberian theorems in probability theory. Nieuw. Arch. Wisk. (4) $3 \cdot 2143-149$.

Bingham, N.H. (1988). Moving averages, in: Almost everywhere convergence I. Academic Press. 131-145.

Bingham, N.H. and Goldie, C.M. (1983). On one-sided Tauberian conditions. Analysis. 3, $159-188$.

Bingham, N.H. and Goldie, C.M. (1983). Riesz means and self-neglecting functions. Math. Z. 199, 443-454.

Bingham, N.H. and MafJima, M. (1985). Summability methods and almost sure convergence. Z. Wahrscheinlichkeitstheorie verw. Geb. 68, 383-392.

Bingham, N.H. and StaDtmüller, U. (1990). Jakimovski methods and almost sure convergence, in: Disorder in Physical Systems. Grimmett, Welsh Eds.; Clarendon Press, Oxford. 5-17.

Bingham, N.H. and Tenenbaum, G. (1986). Riesz and Valiron means and fractional moments. Math. Proc. Cambridge Philos. Soc. 99, 143-149.

Bingham, N.H., Goldie, C.M. and Teugels, J.L. (1987). Regular variation. Cambridge University Press.

CHow, Y.S. (1973). Delayed sums and Borel summability of independent, identically distributed random variables. Bull. Inst. Math. Acad. Sinica. 1, 207-220.

Csörgö, M. and Steinebach, J. (1981). Improved Erdös-Rényi and strong approximation laws for increments of partial sums. Ann. Probab. 9, 988-996.

Deheuvels, P. and Devroye, L. (1987). Limit laws of Erdös-Rényi-Shepp type. Ann. Probab. 15, 1363-1386.

ERDös, P. and RÉnYi, A. (1970). On a new law of large numbers. J. Anal. Math. 23, $103-111$.

FELLER, W. (1968). An extension of the law of the iterated logarithm to variables without variance. J. Math. Mech. 18, 343-355.

Gantert, N. (1996). Large deviations for a heavy-tailed mixing sequence. Preprint.

GRIfFIn, P.S. (1988). The influence of extremes on the law of the iterated logarithm. Probab. Theory Related Fields. 77, 241-270.

GRIFFIN, P.S. (1988a). Non-classical law of the iterated logarithm behaviour for trimmed sums. Probab. Theory Related Fields. 78, 293-319.

Kiesel, R. (1993). General Nörlund Transforms and Power series Methods. Math. Z. 214, $273-286$.

Kiesel, R. (1993a). Power series methods and almost sure covergence. Math. Proc. Cambridge Philos. Soc. 113, 195-205. 
Kiesel, R. and Stadtmüller, U. (1996). Erdös-Rényi-Shepp-Laws and weighted sums of i.i.d. random variables. J. Theoret. Probab. 6, 961-982.

Kolmogoroff, A.N. (1930). Sur la loi forte des grands nombres. C. R. Acad. Sci. Paris. 191, 910-912.

KolmogorofF, A.N. (1933). Grundbegriffe der Wahrscheinlichkeitsrechnung. Springer Verlag.

Komlós, J., Major, P. and Tusnády, G. (1975). An approximation of partial sums of independent rv's and the sample df. I. Z. Wahrscheinlichkeitstheorie verw. Geb. 32, $111-131$.

Komlós, J., Major, P. and Tusnády, G. (1976). An approximation of partial sums of independent rv's and the sample df. II. Z. Wahrscheinlichkeitstheorie verw. Geb. 34, $33-58$.

LAI, T.L. (1974). Summability methods for independent, identically distributed random variables. Proc. Amer. Math. Soc. 45, 253-261.

Lai, T.L. (1974a). Limit theorems for delayed sums. Ann. Probab. 2, 432-440.

MAJor, P. (1976). The approximation of partial sums of independent rv's. Z. Wahrscheinlichkeitstheorie verw. Geb. 35, 213-220.

MORI, T. (1976); The strong law of large numbers when extreme terms are excluded from sums. Z. Wahrscheinlichkeitstheorie verw. Geb. 36, 189-194.

Mori, T. (1977); Stability for sums of i.i.d. random variables when extreme terms are excluded. Z. Wahrscheinlichkeitstheorie verw. Geb. 40, 159-167.

Nagaev, S.V. (1979). Large deviations of sums of independent random variables. Ann. Probab. 7, 745-789.

Shao, Q.-M. (1989). On a problem of Csörgö and Révész. Ann. Probab. 17(2), 809-812.

ShePP, L.A. (1964). A limit law concerning moving averages. Ann. Math. Stat. 35(1), $424-428$.

Stadtmüller, U. (1995). On a family of summability methods and one-sided tauberian conditions. J. Math. Anal. Appl. 196, 99-119.

Steinebach, J. (1978). On a necessary condition for the Erdös-Rényi law of large numbers. Proc. Amer. Math. Soc. 68, 97-100.

Universität Ulm, Abteilung Mathematik III, D-89069 Ulm, Germany. E-mail: lanzinge@mathematik.uni-ulm.de. 